\title{
Local Realistic Interpretation of Entangled Photon Pairs in the Weyl-Wigner Formalism
}

\author{
Emilio Santos* \\ Departamento de Física Moderna, Universidad de Cantabria, Santander, Spain
}

A polarization correlation experiment with two maximally entangled photons created by spontaneous parametric down-conversion is studied in the Weyl-Wigner formalism, that reproduces the quantum predictions. A realistic stochastic interpretation is proposed suggesting that an analysis of the experiments more detailed than the Bell approach may be compatible with local realism. Entanglement appears as a correlation between fluctuations of signal and vacuum fields.

Keywords: local realism, bell inequalities, entangled photons, parametric down-conversion, Weyl-Wigner

\section{OPEN ACCESS}

Edited by:

Ana Maria Cetto,

National Autonomous University of

Mexico, Mexico

Reviewed by:

Herman Batelaan,

University of Nebraska-Lincoln,

United States

Anouar Ben Mabrouk,

University of Kairouan, Tunisia

Kaled Dechoum,

Universidade Federal Fluminense,

Brazil

*Correspondence:

Emilio Santos

emilio.santos@unican.es

Specialty section: This article was submitted to Mathematical Physics, a section of the journal

Frontiers in Physics

Received: 05 March 2020 Accepted: 30 April 2020

Published: 03 June 2020

Citation:

Santos E (2020) Local Realistic Interpretation of Entangled Photon

Pairs in the Weyl-Wigner Formalism. Front. Phys. 8:191

doi: 10.3389/fphy.2020.00191

\section{THE EMPIRICAL REFUTATION OF BELL'S LOCAL REALISM}

In 2015, experiments were reported showing for the first time the loophole-free violation of a Bell inequality $[1,2]$. The result has been interpreted as the "death by experiment for local realism," this being the hypothesis that "the world is made up of real stuff, existing in space and changing only through local interactions... about the most intuitive scientific postulate imaginable" [3]. This statement, and many similar ones, emphasize both the relevance of local realism for our understanding of the physical world and the fact that it has been refuted empirically. Nevertheless, it is worth studying the possibility of a loophole in the empirical refutation via a new definition of locality weaker than Bell's. In this article I search for such a weak locality, compatible with the said experiments [1,2], that involved photon pairs entangled in polarization produced via spontaneous parametric down conversion. Thus I will analyze such experiments using the Weyl-Wigner formalism of quantum optics, rather than the more usual Hilbert-space formalism. Previously I revisit briefly the origin and meaning of the Bell inequalities [4].

Bell defined "local hidden variables" model, later named "local realistic," to be any model of an experiment where the results of all correlation measurements may be interpreted according to the formulas

$$
\begin{aligned}
\langle A\rangle & =\int \rho(\lambda) d \lambda M(\lambda, A),\langle B\rangle=\int \rho(\lambda) d \lambda M(\lambda, B), \\
\langle A B\rangle & =\int \rho(\lambda) d \lambda M(\lambda, A) M(\lambda, B),
\end{aligned}
$$

where $\lambda \in \Lambda$ is one or several random ("hidden") variables, $\langle A\rangle,\langle B\rangle$, and $\langle A B\rangle$ being the expectation values of the results of measuring the observables $A, B$ or their product $A B$, respectively. Here we will consider that the observables correspond to detection, or not, of some signals (e.g., photons) by two parties named Alice and Bob, attaching the values 1 or 0 to these two possibilities. In this case $\langle A\rangle,\langle B\rangle$ correspond to the single and $\langle A B\rangle$ to the coincidence detection rates respectively. The following mathematical conditions are assumed

$$
\rho(\lambda) \geq 0, \int \rho(\lambda) d \lambda=1, M(\lambda, A) \in\{0,1\}, M(\lambda, B) \in\{0,1\} .
$$


Equation (2) corresponds to a "deterministic model" where the statistical aspects derive from the probabilistic nature of the hidden random variables $\{\lambda\}$. More general models may be constructed where the whole interval $[0,1]$ is substituted for $\{0,1\}$ in Equation (2). A constraint of locality is included, namely $M(\lambda, A)$ is independent of $M(\lambda, B)$ and $\rho(\lambda)$ independent of both $M(\lambda, A)$ and $M(\lambda, B)[5]$. From these conditions it is possible to derive empirically testable (Bell) inequalities [6, 7]. The tests are most relevant if the measurements performed by Alice and Bob are specially separated in the sense of relativity theory.

In the following sections, I shall shortly review the treatment within the Weyl-Wigner formalism of the polarization correlation measurement of two maximally entangled photons produced via spontaneous parametric down conversion (SPDC). Thus, I continue a theoretical interpretation of SPDC experiments within the WW formalism in the Heisenberg picture, that was initiated in the nineties of the past century [8-18]. In many of those early studies the approach was heuristic and one of the purposes of this article is to provide a more formal foundation. The $\mathrm{WW}$ formalism suggests an intuitive picture for photon entanglement and the interpretation of SPDC experiments in terms of random variables and stochastic processes. However, there are difficulties with the picture that will be discussed in section 4 below.

\section{THE WEYL-WIGNER FORMALISM IN QUANTUM OPTICS}

\subsection{Definition}

The WW formalism was developed for non-relativistic quantum mechanics, where the basic observables involved are positions, $\hat{x}_{j}$, and momenta, $\hat{p}_{j}$, of the particles [19-25]. It may be trivially extended to quantum optics provided we interpret $\hat{x}_{j}$ and $\hat{p}_{j}$ to be the sum and the difference of the creation, $\hat{a}_{j}^{\dagger}$, and annihilation, $\hat{a}_{j}$, operators of the $j$ normal mode of the radiation. That is

$$
\begin{aligned}
\hat{x}_{j} & \equiv \frac{c}{\sqrt{2} \omega_{j}}\left(\hat{a}_{j}+\hat{a}_{j}^{\dagger}\right), \hat{p}_{j} \equiv \frac{i \hbar \omega_{j}}{\sqrt{2} c}\left(\hat{a}_{j}-\hat{a}_{j}^{\dagger}\right) \\
& \Rightarrow \hat{a}_{j}=\frac{1}{\sqrt{2}}\left(\frac{\omega_{j}}{c} \hat{x}_{j}+\frac{i c}{\hbar \omega_{j}} \hat{p}_{j}\right), \hat{a}_{j}^{\dagger}=\frac{1}{\sqrt{2}}\left(\frac{\omega_{j}}{c} \hat{x}_{j}-\frac{i c}{\hbar \omega_{j}} \hat{p}_{j}\right) .
\end{aligned}
$$

Here $\hbar$ is Planck constant, $c$ the velocity of light and $\omega_{j}$ the frequency of the normal mode. In the following, I will use units $\hbar=c=1$. For the sake of clarity I shall represent the operators in a Hilbert space with a "hat," e.g., $\hat{a}_{j}, \hat{a}_{j}^{\dagger}$, and the amplitudes in the WW formalism without "hat," e.g., $a_{j}, a_{j}^{*}$.

The connection with the Hilbert-space formalism is made via the Weyl transform as follows. For any trace class operator $\hat{M}$ of the former we define its Weyl transform to be a function of the field operators $\left\{\hat{a}_{j}, \hat{a}_{j}^{\dagger}\right\}$, that is

$$
\begin{aligned}
W_{\hat{M}}= & \frac{1}{\left(2 \pi^{2}\right)^{n}} \prod_{j=1}^{n} \int_{-\infty}^{\infty} d \lambda_{j} \int_{-\infty}^{\infty} d \mu_{j} \exp \left[-2 i \lambda_{j} \operatorname{Re} a_{j}-2 i \mu_{j} \operatorname{Im} a_{j}\right] \\
& \times \operatorname{Tr}\left\{\hat{M} \exp \left[i \lambda_{j}\left(\hat{a}_{j}+\hat{a}_{j}^{\dagger}\right)+i \mu_{j}\left(\hat{a}_{j}-\hat{a}_{j}^{\dagger}\right)\right]\right\} .
\end{aligned}
$$

The transform is invertible that is

$$
\begin{aligned}
\hat{M}= & \frac{1}{\left(2 \pi^{2}\right)^{2 n}} \prod_{j=1}^{n} \int_{-\infty}^{\infty} d \lambda_{j} \int_{-\infty}^{\infty} d \mu_{j} \exp \left[i \lambda_{j}\left(\hat{a}_{j}+\hat{a}_{j}^{\dagger}\right)+i \mu_{j}\left(\hat{a}_{j}-\hat{a}_{j}^{\dagger}\right)\right] \\
& \times \prod_{j=1}^{n} \int_{-\infty}^{\infty} d \operatorname{Re} a_{j} \int_{-\infty}^{\infty} d \operatorname{Im} a_{j} W_{\hat{M}}\left\{a_{j}, a_{j}^{*}\right\} \exp \left[-2 i \lambda_{j} \operatorname{Re} a_{j}-2 i \mu_{j} \operatorname{Im} a_{j}\right] .
\end{aligned}
$$

The transform is linear, that is if $f$ is the transform of $\hat{f}$ and $g$ the transform of $\hat{g}$, then the transform of $\hat{f}+\hat{g}$ is $f+g$.

It is standard wisdom that the WW formalism is unable to provide any intuitive picture of the quantum phenomena. The reason is that the Wigner function, that may represent a quantum state, is not positive definite in general and therefore cannot be interpreted as a probability distribution (of positions and momenta in quantum mechanics, or field amplitudes in quantum optics). However, we shall see that in quantum optics the formalism in the Heisenberg representation, where the evolution goes in the field amplitudes, allows the interpretation of the experiments using the Wigner function only for the vacuum state, that is positive definite.

The use of the WW formalism in quantum optics has the following features in comparison with the Hilbert-space formalism:

1. It is just quantum optics, therefore the predictions for experiments are the same.

2. The calculations using the WW formalism are generally no more involved than the corresponding ones in Hilbert space, and sometimes might be easier because no problem of noncommutativity arises.

3. The formalism suggests a physical picture in terms of random variables and stochastic processes. In particular the counterparts of creation and annihilation operators look like random amplitudes.

Here we shall use the formalism in the Heisenberg picture, where the evolution appears in the observables. On the other hand the concept of photon, as a particle, does not appear in the WW formalism.

\subsection{Properties}

All properties of the WW transform in particle systems may be translated to quantum optics via Equation (3). The transform allows getting a function of (c-number) amplitudes for any traceclass operator (e.g., any function of the creation and annihilation operators of "photons"). In particular we may get the (Wigner) function corresponding to any quantum state. For instance the vacuum state, represented by the density matrix $|0\rangle\langle 0|$, is associated with the following Wigner function 


$$
W_{0}=\prod_{j} \frac{2}{\pi} \exp \left(-2\left|a_{j}\right|^{2}\right)
$$

This function might be interpreted as a (positive) probability distribution. Hence the picture that emerges is that the quantum vacuum of the electromagnetic field (also named zeropoint field, $Z P F)$ consists of stochastic fields with a probability distribution independent for every mode, having a Gaussian distribution with mean energy $\frac{1}{2} \hbar \omega$ per mode.

Similarly there are functions associated with the observables. For instance the following Weyl transforms are obtained

$$
\begin{aligned}
\hat{a}_{j} & \leftrightarrow a_{j}, \hat{a}_{j}^{\dagger} \leftrightarrow a_{j}^{*}, \frac{1}{2}\left(\hat{a}_{j}^{\dagger} \hat{a}_{j}+\hat{a}_{j} \hat{a}_{j}^{\dagger}\right) \leftrightarrow a_{j} a_{j}^{*}=\left|a_{j}\right|^{2}, \\
\hat{a}_{j}^{\dagger} \hat{a}_{j} & =\frac{1}{2}\left(\hat{a}_{j}^{\dagger} \hat{a}_{j}+\hat{a}_{j} \hat{a}_{j}^{\dagger}\right)+\frac{1}{2}\left(\hat{a}_{j}^{\dagger} \hat{a}_{j}-\hat{a}_{j} \hat{a}_{j}^{\dagger}\right) \leftrightarrow\left|a_{j}\right|^{2}-\frac{1}{2}, \\
\left(\hat{a}_{j}^{\dagger}+\hat{a}_{j}\right)^{n} & \leftrightarrow\left(a_{j}+a_{j}^{*}\right)^{n},\left(\hat{a}_{j}^{\dagger}-\hat{a}_{j}\right)^{n} \leftrightarrow\left(a_{j}-a_{j}^{*}\right)^{n}, n \text { an integer. }
\end{aligned}
$$

I stress that the quantities $a_{j}$ and $a_{j}^{*}$ are c-numbers and therefore they commute with each other. The former Equation (5) mean that in expressions linear in creation and/or annihilation operator the Weyl transform just implies "removing the hats." However this is not the case in nonlinear expressions in general. In fact from the latter two Equations (5) plus the linearity property it follows that for a product in the WW formalism the Hilbert space counterpart is

$$
a_{j}^{k} a_{j}^{*} \leftrightarrow\left(\hat{a}_{j}^{k} \hat{a}_{j}^{\dagger l}\right)_{s y m}
$$

where the subindex sym means writing the product with all possible orderings and dividing for the number of terms. Hence the WW field amplitudes corresponding to products of field operators may be obtained putting the operators in symmetrical order via the commutation relations. Particular instances that will be useful latter are the following

$$
\begin{gathered}
\hat{a}_{j}^{\dagger} \hat{a}_{j} \rightarrow\left|a_{j}\right|^{2}-\frac{1}{2}, \hat{a}_{j} \hat{a}_{j}^{\dagger} \rightarrow\left|a_{j}\right|^{2}+\frac{1}{2}, \hat{a}_{j}^{2} \rightarrow a_{j}^{2}, \hat{a}_{j}^{\dagger 2} \rightarrow \hat{a}_{j}^{* 2} \\
\hat{a}_{j}^{\dagger} \hat{a}_{j} \hat{a}_{j}^{\dagger} \hat{a}_{j} \rightarrow\left|a_{j}\right|^{4}-\left|a_{j}\right|^{2}, \hat{a}_{j} \hat{a}_{j}^{\dagger} \hat{a}_{j} \hat{a}_{j}^{\dagger} \rightarrow\left|a_{j}\right|^{4}+\left|a_{j}\right|^{2}, \\
\hat{a}_{j}^{\dagger} \hat{a}_{j}^{\dagger} \hat{a}_{j} \hat{a}_{j} \rightarrow\left|a_{j}\right|^{4}-2\left|a_{j}\right|^{2}+\frac{1}{2}, \hat{a}_{j} \hat{a}_{j} \hat{a}_{j}^{\dagger} \hat{a}_{j}^{\dagger} \rightarrow\left|a_{j}\right|^{4}+2\left|a_{j}\right|^{2}+\frac{1}{2} .
\end{gathered}
$$

Other properties may be easily obtained from well known results of the standard Weyl-Wigner formalism in particle quantum mechanics. I will present them omitting the proofs.

Expectation values may be calculated in the WW formalism as follows. In the Hilbert-space formalism they $\operatorname{read} \operatorname{Tr}(\hat{\rho} \hat{M})$, or in particular $\langle\psi|\hat{M}| \psi\rangle$, whence the translation to the WW formalism is obtained taking into account that the trace of the product of two operators becomes

$$
\operatorname{Tr}(\hat{\rho} \hat{M})=\int W_{\hat{\rho}}\left\{\hat{a}_{j}, \hat{a}_{j}^{\dagger}\right\} W_{\hat{M}}\left\{\hat{a}_{j}, \hat{a}_{j}^{\dagger}\right\} \prod_{j} d \operatorname{Re} a_{j} d \operatorname{Im} a_{j} .
$$

That integral is the WW counterpart of the trace operation in the Hilbert-space formalism. Particular instances are the following expectations that will be of interest later on

$$
\begin{aligned}
\left\langle\left|a_{j}\right|^{2}\right\rangle & \equiv \int d \Gamma W_{0}\left|a_{j}\right|^{2}=\frac{1}{2},\left\langle a_{j}^{n} a_{j}^{* m}\right\rangle=0 \text { if } n \neq m . \\
\left\langle 0\left|\hat{a}_{j}^{\dagger} \hat{a}_{j}\right| 0\right\rangle & =\int d \Gamma\left(a_{j}^{*} a_{j}-\frac{1}{2}\right) W_{0}=0, \\
\left\langle 0\left|\hat{a}_{j} \hat{a}_{j}^{\dagger}\right| 0\right\rangle & =\int d \Gamma\left(\left|a_{j}\right|^{2}+\frac{1}{2}\right) W_{0}=2\left\langle\left|a_{j}\right|^{2}\right\rangle=1, \\
\left\langle\left|a_{j}\right|^{4}\right\rangle & =1 / 2,\left\langle\left|a_{j}\right|^{n}\left|a_{k}\right|^{m}\right\rangle=\left\langle\left|a_{j}\right|^{n}\right\rangle\left\langle\left|a_{k}\right|^{m}\right\rangle \text { if } j \neq k .
\end{aligned}
$$

where $W_{0}$ is the Wigner function of the vacuum (Equation 4). This means that in the WW formalism the field amplitude $a_{j}$ (coming from the vacuum) behaves like a complex random variable with Gaussian distribution and mean square modulus $\left\langle\left|a_{j}\right|^{2}\right\rangle=1 / 2$. I point out that the integral for any mode not entering in the function $M\left(\left\{a_{j}, a_{j}^{*}\right\}\right)$ gives unity in the integration due to the normalization of the Wigner function (Equation 4). An important consequence of Equation (8) is that normal (antinormal) ordering of creation and annihilation operators in the Hilbert space formalism becomes subtraction (addition) of $1 / 2$ in the WW formalism. The normal ordering rule is equivalent to subtracting the vacuum contribution.

\subsection{Evolution}

In the Heisenberg picture of the Hilbert-space formalism the density matrix is fixed and any observable, say $\hat{M}$, evolves according to

$$
\frac{d}{d t} \hat{M}=i(\hat{H} \hat{M}-\hat{M} \hat{H}), \hat{M}=\hat{M}(t)
$$

where $\hat{H}$ is the Hamiltonian. Translated to the WW formalism this evolution of the observables is given by the Moyal equation with the sign changed. The standard Moyal equation applies to the evolution of the Wigner function, that represents a quantum state being the counterpart of the density matrix in the Schrödinger picture of the Hilbert space formalism. Thus, in the WW formalism we have

$$
\begin{aligned}
\frac{\partial W_{\hat{M}}}{\partial t}= & 2\left\{\sin \left[\frac{1}{4}\left(\frac{\partial}{\partial \operatorname{Re} a_{j}^{\prime}} \frac{\partial}{\partial \operatorname{Im} a_{j}^{\prime \prime}}-\frac{\partial}{\partial \operatorname{Im} a_{j}^{\prime}} \frac{\partial}{\partial \operatorname{Re} a_{j}^{\prime \prime}}\right)\right]\right. \\
& \left.\times W_{\hat{M}}\left\{a_{j}^{\prime}, a_{j}^{* \prime}, t\right\} H\left(a_{j}^{\prime \prime}, a_{j}^{* \prime \prime}\right)\right\}_{a_{j}},
\end{aligned}
$$

where \{\}$_{a_{j}}$ means making $a_{j}^{\prime}=a_{j}^{\prime \prime}=a_{j}$ and $a_{j}^{* \prime}=a_{j}^{* \prime \prime}=a_{j}^{*}$ after performing the derivatives.

A simple example is the free evolution of the field amplitude of a single mode. The Hamiltonian in the WW formalism may be trivially obtained translating the Hamiltonian of the Hilbertspace formalism, that is

$$
\hat{H}_{\text {free }}=\omega_{j} \hat{a}_{j}^{\dagger} \hat{a}_{j}=\frac{1}{2} \omega_{j}\left(\hat{a}_{j}^{\dagger} \hat{a}_{j}+\hat{a}_{j} \hat{a}_{j}^{\dagger}\right)-\frac{1}{2} \omega_{j}
$$




$$
\rightarrow H_{\text {free }}=\omega_{j}\left(\left|a_{j}\right|^{2}-\frac{1}{2}\right)=\omega_{j}\left[\left(\operatorname{Re} a_{j}\right)^{2}+\left(\operatorname{Im} a_{j}\right)^{2}-\frac{1}{2}\right],
$$

where we have taken the first Equation (8) into account. This leads to

$$
\begin{aligned}
\frac{d}{d t} a_{j} & =\frac{1}{2} \omega_{j}\left[2\left(\operatorname{Im} a_{j}\right)-2\left(\operatorname{Re} a_{j}\right) i\right] \\
& =-i \omega_{j} a_{j} \Rightarrow a_{j}(t)=a_{j}(0) \exp \left(-i \omega_{j} t\right)
\end{aligned}
$$

Another example is the down-conversion process in a nonlinear crystal. Avoiding a detailed study of the physics inside the crystal $[12,26]$ we shall study a single mode problem with the model Hamiltonian[27]

$$
\hat{H}_{I}=A \hat{a}_{s}^{\dagger} \hat{a}_{i}^{\dagger} \exp \left(-i \omega_{P} t\right)+A^{*} \hat{a}_{s} \hat{a}_{i} \exp \left(i \omega_{P} t\right),
$$

when the laser is treated as classically prescribed, undepleted, and spatially uniform field of frequency $\omega_{P}$. The parameter $A$ is proportional to the pump amplitude and the nonlinear susceptibility. In the WW formalism this Hamiltonian becomes (see Equation 5)

$$
H_{I}=A a_{s}^{*} a_{i}^{*} \exp \left(-i \omega_{P} t\right)+A^{*} a_{s} a_{i} \exp \left(i \omega_{P} t\right),
$$

whence taking Equation (9) and (10) into account we have

$$
\begin{aligned}
\frac{d}{d t} a_{s} & =-i \omega_{s} a_{s}-i A a_{i}^{*} \exp \left(-i \omega_{P} t\right), \\
\frac{d}{d t} a_{i} & =-i \omega_{i} a_{i}-i A a_{s}^{*} \exp \left(-i \omega_{P} t\right) .
\end{aligned}
$$

We shall assume that the vacuum field $a_{s}$ evolves as in Equation (10) before entering the crystal and then according to Equation (12) inside the crystal, that is during the time $T$ needed to cross it. In order to get the radiation intensity to second order in $A T \equiv C$ (see below section 2.4) we must solve these two coupled equations also to second order. After some algebra this leads to

$$
\begin{aligned}
a_{s}(t) & =\left(1+\frac{1}{2}|C|^{2}\right) a_{s}(0) \exp \left(-i \omega_{s} t\right)-i C a_{i}^{*}(0) \exp \left[i\left(\omega_{i}-\omega_{P}\right) t\right] \\
& =\left[\left(1+\frac{1}{2}|C|^{2}\right) a_{s}(0)-i C a_{i}^{*}(0)\right] \exp \left(-i \omega_{s} t\right),
\end{aligned}
$$

and the latter equality takes the "energy conservation" into account, that in the WW formalism looks like a condition of frequency matching, $\omega_{P}=\omega_{s}+\omega_{i}$, with no reference to photon energies.

Equation (13) gives the time dependence of the relevant mode of signal after crossing the crystal, but we should take account of the field dependence on position including a factor $\exp \left(i \mathbf{k}_{s} \cdot \mathbf{r}\right)$, that is a phase depending on the path length. Therefore, the correct form of Equation (13) would be, modulo a global phase,

$$
a_{s}(\mathbf{r}, t)=\left[\left(1+\frac{1}{2}|C|^{2}\right) a_{s}(0)-i C a_{i}^{*}(0)\right] \exp \left(i \mathbf{k}_{s} \cdot \mathbf{r}-i \omega_{s} t\right) .
$$

A similar result is obtained for $a_{i}(t)$, that is

$$
a_{i}(\mathbf{r}, t)=\left[\left(1+\frac{1}{2}|C|^{2}\right) a_{i}(0)-i C a_{s}^{*}(0)\right] \exp \left(i \mathbf{k}_{i} \cdot \mathbf{r}-i \omega_{i} t\right) .
$$

Equations (14) and (15) may be interpreted saying that the interaction of the vacuum signal with the pumping laser produces an additional field that travels in the direction of the idler. Similarly the vacuum idler produces a field that travels in the direction of the signal. Therefore, it has sense adding the initial vacuum signal plus the amplification of the idler.

We may perform a change from $C$ to the new parameter $D=\left(1+\frac{1}{2}|C|^{2}\right)^{-1} C$, whence Equation (7) become, to order $O\left(|D|^{2}\right)$,

$$
\begin{aligned}
& E_{s}^{+}=\left(1+\frac{1}{2}|C|^{2}\right)\left[a_{s}+D a_{i}^{*}\right] \exp \left(i \mathbf{k}_{s} \cdot \mathbf{r}-i \omega_{s} t\right), \\
& E_{i}^{+}=\left(1+\frac{1}{2}|C|^{2}\right)\left[a_{i}+D a_{s}^{*}\right] \exp \left(i \mathbf{k}_{i} \cdot \mathbf{r}-i \omega_{i} t\right),|D|<<1,
\end{aligned}
$$

and I will ignore the constant global factor $\left(1+\frac{1}{2}|C|^{2}\right) \sim 1$ because we will be interested in calculating relative detection rates.

Equations (14) and (15), although good enough for calculations, are bad representations of the physics. In fact a physical beam corresponds to a superposition of the amplitudes, $a_{\mathbf{k}}^{*}$, of many modes with frequencies and wavevectors close to $\omega_{s}$ and $\mathbf{k}_{s}$, respectively. For instance we may represent the positive frequency part of the idler beam created in the crystal, to first order in $D$, as follows

$$
\begin{aligned}
E_{i}^{(+)}(\mathbf{r}, t)= & -i D \int f_{i}(\mathbf{k}) d^{3} \mathbf{k} a_{\mathbf{k}}^{*} \exp \left[i\left(\mathbf{k}-\mathbf{k}_{s}\right)\right. \\
& \left.\cdot \mathbf{r}-i\left(\omega-\omega_{s}\right) t\right]+E_{Z P F}^{(+)}
\end{aligned}
$$

where $\omega=\omega(\mathbf{k})$ and $f_{i}(\mathbf{k})$ is an appropriate function, with domain in a region of $\mathbf{k}$ around $\mathbf{k}_{s}$. The field $E_{Z P F}^{(+)}$is the sum of amplitudes of all vacuum modes, including the one represented by $a_{s}$ in Equation (14). (We have neglected a term of order $|C|^{2}$ so that $E_{i}^{(+)}$is correct to order $|C|$ or what is equivalent order $|D|)$. These vacuum modes have fluctuating amplitudes with a probability distribution given by the vacuum Wigner function (Equation 4). It may appear that the amplitude $a_{s}$ is lost "as a needle in the haystack" within the background of many radiation modes, but it is relevant in correlation experiments. In fact the vacuum amplitude $a_{s}$ in Equations (13) or (14) is fluctuating and the same fluctuations appear also in the signal amplitude $a_{s}^{*}$ of Equation (15). Therefore, coincidence counts will be favored when large positive fluctuations of the fields (Equations 13, 15) arrive simultaneously to Alice and Bob detectors. In the Hilbertspace formalism this fact is named "entanglement between a signal and the vacuum." In the WW formalism of quantum optics this entanglement appears as a correlation of fluctuations between a signal and a vacuum field in distant places.

The mentioned properties of the WW formalism are sufficient for the interpretation of experiments involving pure radiation 
field interacting with macroscopic bodies, these defined by their bulk electric properties like the refraction index or the nonlinear electrical susceptibility. Within the WW formalism the interaction between the fields (either signals or vacuum fields) and macroscopic bodies may be treated as in classical electrodynamics. This is for instance the case for the action of a laser on a crystal with nonlinear susceptibility, studied elsewhere $[12,26]$.

\section{PHOTON PAIRS ENTANGLED IN POLARIZATION}

In this section, I will apply the WW formalism to the description of the polarization correlation of entangled photon pairs produced via spontaneous parametric down-conversion (SPDC). I will assume that the experimental set-up is made so that the fields arriving at the detectors correspond to so called "photon pairs maximally entangled" in polarization. These fields are obtained in the outgoing channels of a beam splitter after sending the signal and idler beams produced by SPDC to the incoming channels. The electromagnetic radiation is a vector field with two possible polarizations and I should take into account this fact including vectors in the description, that we have omitted till now. Thus, I will write the beams produced as follows

$$
\begin{aligned}
& \mathbf{E}_{A}^{+}=\left(a_{s}+D a_{i}^{*}\right) \exp \left(-i \omega_{s} t\right) \mathbf{v}+i\left(a_{i}+D a_{s}^{*}\right) \exp \left(-i \omega_{i} t\right) \mathbf{h}, \\
& \mathbf{E}_{B}^{+}=-i\left(a_{s}+D a_{i}^{*}\right) \exp \left(-i \omega_{s} t\right) \mathbf{h}+\left(a_{i}+D a_{s}^{*}\right) \exp \left(-i \omega_{i} t\right) \mathbf{v}
\end{aligned}
$$

where $\mathbf{h}$ is a unit vector horizontal and $\mathbf{v}$ vertical. We have not written explicitly the dependence on position, that could be restored without difficulty (see Equation 17). Furthermore, from now on I will ignore all spacetime dependence that usually contributes phase factors irrelevant for our argument. The complex conjugate of the above fields will be labeled as follows

$$
\left(\mathbf{E}_{A}^{+}\right)^{*} \equiv \mathbf{E}_{A}^{-},\left(\mathbf{E}_{B}^{+}\right)^{*} \equiv \mathbf{E}_{B}^{-}
$$

The "two photons entangled in polarization," represented by Equation (18) in the Weyl-Wigner formalism, will arrive at the Alice and Bob polarization analyzers put at angles $\theta$ and $\phi$ with the vertical, respectively. Hence the beams emerging from them will have field amplitudes

$$
\begin{aligned}
& E_{A}^{+}=\left(a_{s}+D a_{i}^{*}\right) \cos \theta+i\left(a_{i}+D a_{s}^{*}\right) \sin \theta, \\
& E_{B}^{+}=-i\left(a_{s}+D a_{i}^{*}\right) \sin \phi+\left(a_{i}+D a_{s}^{*}\right) \cos \phi,
\end{aligned}
$$

and polarizations at angles $\theta$ and $\phi$ with the vertical, respectively. For later convenience I define the partial fields

$E_{A 0}^{+}=a_{s} \cos \theta+i a_{i} \sin \theta, E_{B 0}^{+}=-i a_{s} \sin \phi+a_{i} \cos \phi$,

$E_{A 1}^{+}=D\left[a_{i}^{*} \cos \theta+i a_{s}^{*} \sin \theta\right], E_{B 1}^{+}=D\left[-i a_{i}^{*} \sin \phi+a_{s}^{*} \cos \phi\right]$.

The single, $P_{A}, P_{B}$, and coincidence, $P_{A B}$, detection rates in the $\mathrm{WW}$ formalism may be obtained by comparison with the rates calculated in the Hilbert-space formalism. Thus, in the following we revisit briefly the Hilbert-space treatment of the entangled photon pairs. I will start with the quantum fields arriving at Alice and Bob, respectively, that are the counterparts of the WW (Equation 19). It is trivial to get them either from Equation (11) or, taking Equation (5) into account, that is "putting hats" in the WW (Equation 20). We get the field operators

$$
\begin{aligned}
\hat{E}_{A}^{+} & =\hat{E}_{A 0}^{+}+\hat{E}_{A 1}^{+}, \hat{E}_{B}^{+}=\hat{E}_{B 0}^{+}+\hat{E}_{B 1}^{+}, \\
\hat{E}_{A 0}^{+} & =\hat{a}_{s} \cos \theta+i \hat{a}_{i} \sin \theta, \hat{E}_{B 0}^{+}=-i \hat{a}_{s} \sin \phi+\hat{a}_{i} \cos \phi, \\
\hat{E}_{A 1}^{+} & =D\left[\hat{a}_{i}^{\dagger} \cos \theta+i \hat{a}_{s}^{\dagger} \sin \theta\right], \hat{E}_{B 1}^{+}=D\left[-i \hat{a}_{i}^{\dagger} \sin \phi+\hat{a}_{s}^{\dagger} \cos \phi\right],
\end{aligned}
$$

and similar for the Hermitian conjugates. Alice's single detection rate is proportional to the following vacuum expectation (with $\left.\hat{E}_{A}^{-}=\left(\hat{E}_{A}^{+}\right)^{\dagger}\right)$

$$
\begin{aligned}
P_{A} & =\left\langle 0\left|\hat{E}_{A}^{-} \hat{E}_{A}^{+}\right| 0\right\rangle=|D|^{2}\left\langle 0\left|\hat{E}_{A 1}^{-} \hat{E}_{A 1}^{+}\right| 0\right\rangle \\
& =|D|^{2}\left\langle 0\left|\left(\hat{a}_{i} \cos \theta-i \hat{a}_{s} \sin \theta\right)\left(\hat{a}_{i}^{\dagger} \cos \theta+i \hat{a}_{s}^{\dagger} \sin \theta\right)\right| 0\right\rangle \\
& =|D|^{2}\left\langle 0\left|\hat{a}_{i} \hat{a}_{i}^{\dagger} \cos ^{2} \theta+\hat{a}_{s} \hat{a}_{s}^{\dagger} \sin ^{2} \theta\right| 0\right\rangle=|D|^{2},
\end{aligned}
$$

where in the former equality, I have neglected creation $\hat{E}_{A 0}^{-}$ (annihilation $\hat{E}_{A 0}^{+}$) operators appearing on the left (right). A similar result may be obtained for the single detection rate of Bob, that is

$$
P_{B}=\left\langle 0\left|\hat{E}_{B}^{-} \hat{E}_{B}^{+}\right| 0\right\rangle=|D|^{2}, \hat{E}_{B}^{-}=\left(\hat{E}_{B}^{+}\right)^{\dagger}
$$

We are assuming ideal detectors, but for real detectors $P_{A}$ and $P_{B}$ should be multiplied times the detection efficiencies $\eta_{A}$ and $\eta_{B}$, and the coincidence rate $P_{A B}$ times $\eta_{A} \eta_{B}$.

In order to get the detection rule for single rates in the $\mathrm{WW}$ formalism we should translate Equation (22) taking Equation (8) into account. We get

$$
\begin{aligned}
P_{A}= & {\left[\left(\left\langle\left|a_{i}\right|^{2}\right\rangle-\frac{1}{2}\right) \cos ^{2} \theta+\left(\left\langle\left|a_{s}\right|^{2}\right\rangle-\frac{1}{2}\right) \sin ^{2} \theta\right] } \\
& \left.+|D|^{2}\left[\left(\left\langle\left|a_{s}\right|^{2}\right\rangle+\frac{1}{2}\right) \cos ^{2} \theta+\left(\left.|| a_{i}\right|^{2}\right\rangle+\frac{1}{2}\right) \sin ^{2} \theta\right] \\
= & |D|^{2}\left[\cos ^{2} \theta+\sin ^{2} \theta\right]=|D|^{2},\left\langle a_{i} a_{s}^{*}\right\rangle=\left\langle a_{s} a_{i}^{*}\right\rangle=0,
\end{aligned}
$$

that agrees with the result calculated in the Hilbert-space formalism (Equation 22). Now we compare Equation (24) with the average of the field intensity arriving at Alice (see Equation 19), that is

$$
\begin{aligned}
\left\langle I_{A}\right\rangle= & \left\langle\left|E_{A}^{+}\right|^{2}\right\rangle=\left\langle\left|a_{i}\right|^{2}\right\rangle \cos ^{2} \theta+\left\langle\left|a_{s}\right|^{2}\right\rangle \sin ^{2} \theta \\
& +|D|^{2}\left[\left\langle\left|a_{s}\right|^{2}\right\rangle \cos ^{2} \theta+\left\langle\left|a_{i}\right|^{2}\right\rangle \sin ^{2} \theta\right]=\frac{1}{2}\left(1+|D|^{2}\right) .
\end{aligned}
$$

We see that going from Equation (26) to Equation (24) the signal terms (those of order $|D|^{2}$ ) are multiplied times 2, whilst those coming from the vacuum (of order unity) are eliminated. This may be seen as a subtraction of the vacuum $(\mathrm{ZPF})$ and 
multiplication of the signal times 2, which leads to the following rule for the single detection rate in the $\mathrm{WW}$ formalism:

$$
P_{A}=2\left\langle I_{A}\right\rangle-2\left\langle I_{A 0}\right\rangle, I_{A}=E_{A}^{+} E_{A}^{-}=\left|E_{A}^{+}\right|^{2}, I_{A 0}=\left|E_{A 0}^{+}\right|^{2} .
$$

The Hilbert-space rule for the coincidence rate is the vacuum expectation value of the product of four field operators in normal order. Here we have two terms

$$
P_{A B}=\frac{1}{2}\left\langle 0\left|\hat{E}_{A}^{-} \hat{E}_{B}^{-} \hat{E}_{B}^{+} \hat{E}_{A}^{+}\right| 0\right\rangle+\frac{1}{2}\left\langle 0\left|\hat{E}_{B}^{-} \hat{E}_{A}^{-} \hat{E}_{A}^{+} \hat{E}_{B}^{+}\right| 0\right\rangle,
$$

that would be equal if $\hat{E}_{A}^{+}$and $\hat{E}_{B}^{+}$commute. The former expectation may be evaluated to order $|D|^{2}$ as follows

$$
\begin{aligned}
\langle 0 & \left.\left|\hat{E}_{A}^{-} \hat{E}_{B}^{-} \hat{E}_{B}^{+} \hat{E}_{A}^{+}\right| 0\right\rangle=\left\langle 0\left|\hat{E}_{A 1}^{-} \hat{E}_{B}^{-} \hat{E}_{B}^{+} \hat{E}_{A 1}^{+}\right| 0\right\rangle=\left\langle 0\left|\hat{E}_{A 1}^{-} \hat{E}_{B 0}^{-} \hat{E}_{B 0}^{+} \hat{E}_{A 1}^{+}\right| 0\right\rangle \\
& =\left\langle 0\left|\hat{E}_{A 1}^{-} \hat{E}_{B 0}^{-}\right| 0\right\rangle\left\langle 0\left|\hat{E}_{B 0}^{+} \hat{E}_{A 1}^{+}\right| 0\right\rangle=\left|\left\langle 0\left|\hat{E}_{B 0}^{+} \hat{E}_{A 1}^{+}\right| 0\right\rangle\right|^{2} \\
& =|D|^{2}\left|\left\langle 0\left|\left(-i \hat{a}_{s} \sin \phi+\hat{a}_{i} \cos \phi\right)\left(\hat{a}_{i}^{\dagger} \cos \theta+i \hat{a}_{s}^{\dagger} \sin \theta\right)\right| 0\right\rangle\right|^{2},
\end{aligned}
$$

where the former equality, similar to Equation (22), removes creation operators on the left and annihilation operators on the right, the second one removes terms of order $|D|^{4}$ and the rest is trivial. The latter term of Equation (27) gives a similar contribution so that we get

$$
\begin{aligned}
P_{A B} & =\frac{1}{2}\left|\left\langle 0\left|\hat{E}_{B 0}^{+} \hat{E}_{A 1}^{+}\right| 0\right\rangle\right|^{2}+\frac{1}{2}\left|\left\langle 0\left|\hat{E}_{A 0}^{+} \hat{E}_{B 1}^{+}\right| 0\right\rangle\right|^{2} \\
& =|D|^{2} \cos ^{2}(\theta-\phi) .
\end{aligned}
$$

Here the creation operators are placed to the right and those of annihilation to the left, so that no subtraction is required in passing to the WW formalism. It is enough to substitute c-number amplitudes multiplied times 2 for the field operators, in order to get the rule for the coincidence rate in the $\mathrm{WW}$ formalism. That is

$$
\begin{aligned}
\left\langle E_{A 0}^{+} E_{B 1}^{+}\right\rangle+\left\langle E_{A 1}^{+} E_{B 0}^{+}\right\rangle & =\left\langle E_{A}^{+} E_{B}^{+}\right\rangle=D \cos (\theta-\phi) \\
& \Rightarrow P_{A B}=\left|\left\langle E_{A}^{+} E_{B}^{+}\right\rangle\right|^{2}=|D|^{2} \cos ^{2}(\theta-\phi),
\end{aligned}
$$

where we have taken Equations (20) and (8) into account. Here the vacuum subtraction is not explicit because the vacuum term would be zero, that is $\left\langle E_{A 0}^{+} E_{B 0}^{+}\right\rangle=0$.

It is interesting to get the coincidence detection rate in terms of field intensities, rather than amplitudes. To do that we start calculating

$$
\left\langle I_{A} I_{B}\right\rangle=\left\langle E_{A}^{+} E_{A}^{-} E_{B}^{+} E_{B}^{-}\right\rangle .
$$

In the WW formalism the field amplitudes are c-numbers, therefore they commute, and the averages should be performed as in Equation (8). The expectation (Equation 30) may be obtained taking into account that the fields have the mathematical properties of Gaussian random variables (see Equation 4) (although this section is devoted to calculations and for the moment I am not committed to any physical interpretation). Thus, I apply a well-known property of the average of the product of four Gaussian random variables, that is

$$
\begin{aligned}
\left\langle I_{A} I_{B}\right\rangle & =\left\langle E_{A}^{+} E_{A}^{-}\right\rangle\left\langle E_{B}^{+} E_{B}^{-}\right\rangle+\left\langle E_{A}^{+} E_{B}^{-}\right\rangle\left\langle E_{A}^{-} E_{B}^{+}\right\rangle+\left\langle E_{A}^{+} E_{B}^{+}\right\rangle\left\langle E_{A}^{-} E_{B}^{-}\right\rangle \\
& =\left\langle I_{A}\right\rangle\left\langle I_{B}\right\rangle+\left|\left\langle E_{A}^{+} E_{B}^{-}\right\rangle\right|^{2}+\left|\left\langle E_{A}^{+} E_{B}^{+}\right\rangle\right|^{2}
\end{aligned}
$$

A similar procedure but involving the vacuum intensities, gives

$$
\left\langle I_{A 0} I_{B 0}\right\rangle=\left\langle I_{A 0}\right\rangle\left\langle I_{B 0}\right\rangle+\left|\left\langle E_{A 0}^{+} E_{B 0}^{-}\right\rangle\right|^{2}+\left|\left\langle E_{A 0}^{+} E_{B 0}^{+}\right\rangle\right|^{2} .
$$

Here the third term does not contribute and the second one equals the second term of Equation (31) to order $|D|^{2}$. Hence, we get the rule for the coincidence rate in the WW formalism that in the following I write both in terms of fields and in terms of intensities.

$$
P_{A B}=\left|\left\langle E_{A}^{+} E_{B}^{+}\right\rangle\right|^{2}=\left\langle I_{A} I_{B}\right\rangle-\left\langle I_{A}\right\rangle\left\langle I_{B}\right\rangle-\left\langle I_{A 0} I_{B 0}\right\rangle+\left\langle I_{A 0}\right\rangle\left\langle I_{B 0}\right\rangle .
$$

\section{LOCALITY IN THE EXPERIMENTS WITH ENTANGLED PHOTON PAIRS}

\subsection{Realistic Interpretation of the Vacuum Radiation Field}

I emphasize again that the WW formalism provides an alternative formulation of quantum optics, physically equivalent to the more common Hilbert-space. But it suggests a picture of the optical phenomena quite different from the latter, where photon is the fundamental concept. Indeed the WW picture may provide a local realistic interpretation in terms of random variables and stochastic processes. In the following, I present the main ideas of this interpretation. It rests upon several assumptions as follows.

The fundamental hypothesis is that the electromagnetic vacuum field is a real stochastic field (the zeropoint field, ZPF). If expanded in normal modes the ZPF has a (positive) probability distribution of the amplitudes given by Equation (4). According to that assumption any photodetector would be immersed in an extremely strong radiation, infinite if no cut-off existed. Thus, how might we explain that detectors are not activated by the vacuum radiation? Firstly the strong vacuum field is effectively reduced to a weaker level if we assume that only radiation within some (small) frequency interval is able to activate a photodetector, that is the interval of sensitivity $\left(\omega_{1}, \omega_{2}\right)$. However, the problem is not yet solved because signals involved in experiments have typical intensities of order the vacuum radiation in the said frequency interval so that the detector would be unable to distinguish a signal from the ZPF noise. Our proposal is to assume that a detector may be activated only when the net Poynting vector (i.e., the directional energy flux) of the incoming radiation is different from zero, including both signal and vacuum fields. More specifically I will assume that the detector possesses an active area and the probability of a photocount is proportional to the net radiant energy flux crossing that area from the front side during some activation time, the probability being zero if the net flux crossing the area is in the reverse direction. 
These assumptions allow to understand qualitatively why the signals, but not the vacuum fields, activate detectors. Indeed the $\mathrm{ZPF}$ arriving at any point (in particular the detector) would be isotropic on the average, whence its associated mean Poynting vector would be nil, therefore only the signal radiation should produce photocounts. A problem remains because the vacuum fields are fluctuating so that the Poynting vector also fluctuates. Hence we may predict the existence of some dark rate even at zero Kelvin. The problems derived from the fluctuations diminish taking into account that photocounts are not produced by an instantaneous interaction of the fields with the detectors but the activation requires some time interval, a known fact in experiments. Therefore the effective activating energy flux is a time average whose fluctuations are plausibly small.

Of course these arguments are qualitative and a quantitative estimate should be worthwhile. However making such an estimate is rather involved and in the following I will just sketch the steps required. We should start calculating the probability distribution of the Poynting vector $\mathbf{P}$ due to the ZPF at a point $\mathbf{r}$ in space at time $t$. If we expand the ZPF in plane waves as usual, the probability of the $\mathbf{Z}$ component of $\mathbf{P}$ may be written as a sum involving all radiation modes, that is

$$
\operatorname{Prob}\left(P_{z}\right)=\left[\sum_{j} \sum_{k} a_{j} a_{k} \operatorname{Prob}\left(a_{j}, a_{k}\right) \mathbf{E}_{j}(\mathbf{r}, t) \times \mathbf{B}_{k}(\mathbf{r}, t)\right] \cdot \mathbf{u}_{z},
$$

where $a_{j}\left(a_{k}\right)$ is the amplitude of the mode $j(k), \mathbf{E}_{j}\left(\mathbf{B}_{k}\right)$ its associated electric (magnetic) field and $\mathbf{u}_{z}$ is a unit vector in the $\mathrm{Z}$ direction. The probability density $\operatorname{Prob}\left(a_{j}, a_{k}\right)$ is related to Equation (4). We assume that only field components with frequencies in the sensitivity interval $\left(\omega_{1}, \omega_{2}\right)$ are effective for detection, whence we should restrict the double sum to modes with frequencies in that interval. Hence we might obtain the probability distribution of the integrated energy flux, $\Phi$, crossing the active area of the detector during the activation time $T$. Finally we may assume that a detection event takes place whenever $\Phi$ surpasses a threshold $\Phi_{0}>0$ related to the detection efficiency, $\eta$, of the detector. If the ZPF is isotropic the flux crossing the active area of the detector might be positive or negative with equal probabilities but the ratio $\Phi / T$, would be smaller as $T$ is larger, zero for $T \rightarrow \infty$. We may choose $\Phi_{0}$, as a function of $T$, so that the ZPF $\Phi$ surpasses $\Phi_{0}$ very rarely. However for any finite $T$ there will be a finite probability that $\Phi>\Phi_{0}$ thus producing photocounts even in the absence of signals. These spurious counts give a dark rate $r$ usually attributed to thermal fluctuations. Indeed the experimental dark rate is known to decrease with temperature, but we propose that $r$ would remain finite at zero Kelvin. Now we study the case when there is a signal, superimposed to the $\mathrm{ZPF}$, arriving at the detector. The signal may be weak with respect to a typical short-time fluctuation of the ZPF, but it is positive at all times because signals arrive at the detector in the positive $\mathrm{Z}$ direction. Thus, a positive quantity should be added to the fluctuating energy flux due to the ZPF, calculated via Equation (34). In a particular experiment we should choose $T$, the sensitivity interval and the threshold $\Phi_{0}$ such that we have high detection efficiency $\eta$ and small dark rate $r$, but there are obvious constraints. For instance in order to have a small $r$ we need high $T$ and/or high $\Phi_{0}$, but in this case some signals will become undetected leading to a decrease of $\eta$. I propose that these constraints are the physical reason for the difficulty of manufacturing very efficient photon counters.

\subsection{Interpretation of the Photon Experiments}

Our aim is to achieve a realistic local interpretation of the experiments measuring polarization correlation of entangled photon pairs, that we studied with the WW formalism in the previous section. Thus, I will consider two vacuum beams entering the nonlinear crystal, where they give rise to a "signal" and an "idler" beams. After crossing several appropriate devices they produce fields that will arrive at the Alice and Bob detectors. I do not attempt to present a detailed model, that should involve many radiation modes, in order to represent the signals as (narrow) beams (see Equation 17). I will study only detection rates, that may be illustrated with just 2 vacuum modes.

In agreement with our previous hypotheses a photodetection should derive from a relatively large integrated energy flux crossing the active surfaces. In actual experiments the pumping laser is pulsed and it is a fact that the detection rates are far smaller than the pulsing rate. In our interpretation this means that only in a tiny fraction of laser pulses, the flux $\Phi$ becomes greater than $\Phi_{0}$. Thus, I will replace the threshold assumption of the previous subsection, i.e., detection when $\Phi>\Phi_{0}$, by another assumption, more convenient for the simplicity of calculations, namely that the detection probability is proportional to $\Phi$ if $\Phi>0$, zero otherwise. I believe that the new assumption might be derived from the old one but I omit the proof. In the following it will be convenient to write everything in terms of the Poynting vector $\vec{I}$ rather than the integrated flux, $\Phi$, assuming that the single detection probability per time window, $T$, by Alice is given by

$$
P_{A}=\left\langle\left[M_{A}\right]_{+}\right\rangle, M_{A} \equiv T^{-1} \int_{0}^{T} \vec{n}_{A} \cdot \vec{I}_{\text {total }}^{A}\left(\mathbf{r}_{A}, t\right) d t
$$

where $[M]_{+}=M$ if $M>0,[M]_{+}=0$ otherwise, and $\vec{n}_{A}$ is a unit vector in the direction of the incoming signal beam, assumed normal to the active area of the detector. The experimental time window has a duration of the order of a laser pulse, and may be different from the activation time of the detector but I will use the same label $T$. I shall use units such that both the intensities and the detection rates are dimensionless defining the rate as probability of a photodetection within one time window $T$. In Equation (35), I include a positivity constraint that I will ignore in the following substituting $M_{A}$ for $\left[M_{A}\right]_{+}$. This approximation underestimate the rate. In fact the quantity $M_{A}$ defined in Equation (35) will be positive in a fraction, say $f<1$, of the samples and negative in the fraction $1-f$. In the former case $\left[M_{A}\right]_{+}=M_{A}$, in the latter $\left[M_{A}\right]_{+}=0$. Therefore $\left\langle M_{A}\right\rangle<$ $\left\langle\left[M_{A}\right]_{+}\right\rangle$, but we assume that the error is small if the activation time is large as commented in the previous subsection.

In order to provide a quantitative argument, I will consider a simplified model involving just two radiation modes, $a_{s}$ and 
$a_{i}$, and follow closely the calculation of the section 3 . The fields emerging from the nonlinear crystal, after crossing several appropriate devices, will arrive at the Alice and Bob detectors. Each one of these fields consists of two parts, one large, of order unity and another of order $|D|<<1$. The former, given in Equation (20), is what would arrive at the detectors if there was no pumping laser and therefore no signal. It is just a part of the ZPF, whilst the rest of the ZPF consists of radiation that did not appear explicitly in the equations of section 3 because it was not needed in the calculations. As discussed above the total ZPF should have the property of isotropy, therefore giving an energy flux that may be positive or negative, nil on the average. The terms of order $|D|$, given by Equation (20), correspond to the signal produced in the nonlinear crystal after the modifications introduced by beam splitters and polarizers (and other devices like apertures, filters, lens systems, etc. whose action is not detailed in our simplified model). In summary the Poynting vector of the radiation at the (center of the) active area of Alice's detector may be written

$$
\text { Alice }: \vec{I}_{\text {total }}^{A}(t)=\vec{I}_{Z P F}^{A}(t)+\vec{I}_{A}(t) .
$$

$\vec{I}_{A}$ corresponding to the field $E_{A}$, Equation (19) and $\vec{I}_{Z P F}^{A}(t)$ comes from the rest of the ZPF. $\vec{I}_{A}(t)$ has the direction of $\vec{n}_{A}$, (Equation 35 ), whence the radiation intensity equals the modulus of the Poynting vector. Furthermore, its intensity is time independent (the time dependence of the signal fields derives from the sum of many modes as in Equation 17), so that we may write

$$
M_{A}=I_{Z P F}^{A}+I_{A}, I_{Z P F}^{A} \equiv T^{-1} \int_{0}^{T} \vec{n}_{A} \cdot \vec{I}_{Z P F}^{A}\left(\mathbf{r}_{A}, t\right) d t,
$$

and similar for Bob's $M_{B}$.

In order to get the Alice single detection rate we need the average of $M_{A}$, that we will evaluate by comparison with the case when there is no pumping on the nonlinear crystal. In this case $I_{A}$ becomes the intensity $I_{A 0}$, Equation (26), and the Poynting vector of all vacuum fields arriving at the detector of Alice, i.e., $\vec{I}_{Z P F}^{A}(t)+\vec{I}_{A 0}(t)$, should have nil average due to the isotropy of the $Z P F$. And similar for Bob. As a consequence the intensities $I_{A 0}$ and $I_{B 0}$, Equation (26), should fulfill the following equalities

$$
\left\langle I_{Z P F}^{A}+I_{A 0}\right\rangle=\left\langle I_{Z P F}^{B}+I_{B 0}\right\rangle=0 .
$$

It would appear that this relation could not be true for all values of the angles $\theta, \phi$ (Equation 20) because the ZPF Poynting vector $\hat{I}_{Z P F}^{A}$ and $\hat{I}_{Z P F}^{B}$ should not depend on our choice of angles whilst $I_{A 0}$ and $I_{B 0}$ do depend. However, this is flawed, the positions of the polarizers may influence also the $\mathrm{ZPZ}$ arriving at the detectors and it is plausible that the total Poynting vector has always zero mean. From Equation (35) to (38) we may derive the single rates of Alice and Bob, that is

$$
P_{A}=\left\langle M_{A}\right\rangle=\left\langle I_{A}\right\rangle-\left\langle I_{A 0}\right\rangle, P_{B}=\left\langle M_{B}\right\rangle=\left\langle I_{B}\right\rangle-\left\langle I_{B 0}\right\rangle .
$$

The result agrees with the quantum calculation in the WW formalism except for a factor 2 that derives from a different definition of field amplitudes. I point out that no dark rate appears in Equation (39) due to our approximation $[M]_{+}=M$, see comment after Equation (35).

An analysis should be also made for the coincidence detection rates, but it is more involved and will not be included in the present paper (for a preliminary approach see [28]). A sketch is as follows. The coincidence detection probability in a given time window would be given by an extension of Equation (35), that is

$$
P_{A B}=\left\langle\left[M_{A}\right]_{+}\left[M_{B}\right]_{+}\right\rangle \text {. }
$$

This will give rise to many correlations between the field intensities arriving at Alice and Bob detectors. Determining the correct values of the correlations is a subtle matter, but I hope that an appropriate choice might reproduce the quantum predictions and the empirical results. The relevant achievement in the experiments is the strong correlation, that is the fact that $P_{A B}$ is of the same order as $P_{A}$ and $P_{B}$. Such a strong correlation is crucial for the violation of a Bell inequality. A qualitative argument for that correlation in our model is the following. The vacuum fields arriving at the nonlinear crystal are enhanced by the action of the laser, thus producing the socalled signal and idler fields. That enhancement is rarely strong enough to produce detection events in Alice and Bob detectors. However, from time to time a combination of high values of both relevant (fluctuating) vacuum fields, $a_{s}$ and $a_{i}$, will give a relatively intense signal and idler fields that combined in the form of Equation (18) produce high field intensities arriving at both Alice and Bob detectors, giving rise to a coincidence count. The point is that a detection event requires simultaneous high values of $a_{s}$ and $a_{i}$ and in this case the signal intensities arriving at Alice and Bob are both large, whence detections are most probably produced in coincidence (provided the angles $\theta, \phi$ in Equation 19 are appropriate).

The WW formalism suggests a quite different picture from the Hilbert-space one in terms of photons. We should not assume that the small value of the coupling parameter $|D|^{2}$ implies that the production probability of "entangled photon pairs" during a time window is small, but that the probability of detection, conditional to the photon production, is of the order of unity. (The latter probability is defined as the detection efficiency). In the WW formalism the probability of a photocount by Alice or Bob does not factorize that way. Furthermore, the concept of photon does not appear at all, but there are continuous fluctuating fields including a real $Z P F$ arriving at the detectors that are activated when the arriving signal intensities are large enough.

Finally I stress that the hypothesis that the quantum vacuum fields are real allows a more detailed model of the experiments than Bell's approach and the model is local. Indeed, as in the interpretation of single detections rates (Equation 39), the signals (accompanied by vacuum) travel causally from the source to the detectors.

\section{DISCUSSION}

Bell's work of 1964 put forward an acute conflict in theoretical physics. The derived Bell inequalities are currently seen as 
necessary conditions for any local realistic theory of physics. On the other hand the inequalities are incompatible with some predictions of quantum mechanics. The conclusion is that either local realism does not hold in nature or that quantum mechanics is not valid in all circumstances. Both alternatives are hard to accept.

Realims is a principle that may be stated saying that: In science we ought to be concerned with what nature does, not just with predictions of empirical results, so that we may get a picture of the natural world. In physics the picture should be quantitative, and we should interpret the observed phenomena in terms of general laws. In the microscopic domain the laws are those of quantum mechanics. The problem is that for some experiments, like those mentioned in section 1 [1,2], the evolution of particles and/or fields, seems to require influences traveling at a superluminal velocity. This means a violation of "locality."

Locality (or relativistic causality) was defined and emphatically supported by Einstein. For instance in his authobiographical notes [29] Einstein wrote "On one supposition we should, in my opinion, absolutely hold fast: the real factual situation of the system S2 is independent of what is done with the system $\mathrm{S} 1$, which is spatially separated from the former." Thus, the violation of locality by experiments might look as a contradiction between quantum theory and relativity. The present wisdom is that locality has been indeed violated by the recent experiments but the conflict with relativity is minimized by the fact that quantum mechanics does not predict the possibility of sending superluminal signals (no-signaling theorem). However, in the views of many people including this author, a violation of locality is highly unsatisfactory.

In this paper, I offer a possible solution, namely that the Bell analysis sketched in the Introduction section does not apply to the commented experiments. In fact the standard method to do with a system of entangled photon pairs, or entangled quantum subsystems in general, is to start with the quantum representation of the whole system by a quantum state, that is a vector $|\psi\rangle$ of the Hilbert space. In the case of two photons entangled in polarization a representation of the quantum state may be

$$
|\psi\rangle=\frac{1}{\sqrt{1+|c|^{2}}}(|V(a)\rangle|H(b)\rangle-c|H(a)\rangle|V(b)\rangle),
$$

where $c$ is a (nonzero) complex number, $V(a)(H(a))$ means that Alice's photon has vertical (horizontal) polarization and similar for Bob. Checking that the representation (Equation 40)

\section{REFERENCES}

1. Shalm LK, Meyer-Scott E, Christensen BG, Bierhorst P, Wayne MA, Stevens MJ, et al. A strong loophole-free test of local realism. Phys Rev Lett. (2015) 115:250402. doi: 10.1103/PhysRevLett.115.250402

2. Giustina M, Versteegh MAM, Wengerowsky S, Handsteiner J, Hochrainer A, Phelan K, et al. A significant loophole-free test of Bell's theorem with entangled photons. Phys Rev Lett. (2015) 115:250401. doi: 10.1103/PhysRevLett.115.250401

3. Wiseman H. Quantum physics: death by experiment for local realism. Nature. (2015) 526:649-50. doi: 10.1038/nature15631 is correct is obtained by several measurements performed, in particular the single rates and the coincidence rate by Alice and Bob for detections after the photons cross polarizers at appropriate angles. The relevant result is that those rates violate a Bell inequality for appropriate choices of angles. Thus assuming that Bell analysis was correct (see section 1), people concludes that local realism has been empirically refuted.

Our criticism to that conclusion is that Bell analysis is not valid for the commented experiments. It is necessary to study in detail all elements involved in the production of the entangled "photon" pairs. (Actually we should speak of entangled radiation modes rather than photons). In particular the action of the quantum vacuum electromagnetic radiation. In some sense the $\mathrm{ZPF}$ is taken into account when one studies quantum optics using the standard Hilbert-space formalism in the Heisenberg representation, as revisited in section 2, where a comparison is made with the treatment in the Weyl-Wigner formalism. In the former the quantum vacuum fields are represented by linear combinations of creation and annihilation operators of photons. This abstract treatment leads people to take the vacuum fields as "virtual," that is purely formal devices useful for calculations but devoid of reality. The novelty of the present paper is to assume that the vacuum fields are real stochastic fields, something that appears as quite plausible in the Weyl-Wigner formalism. Thus, I propose that it is possible to interpret the experiments $[1,2]$ assuming that all quantum fields involved, including the vacuum ones, are real fluctuating (stochastic) fields that propagate causally in space.

\section{DATA AVAILABILITY STATEMENT}

The original contributions presented in the study are included in the article/supplementary materials, further inquiries can be directed to the corresponding author/s.

\section{AUTHOR CONTRIBUTIONS}

The author confirms being the sole contributor of this work and has approved it for publication.

\section{ACKNOWLEDGMENTS}

This manuscript has been released as a pre-print at arXiv:1908.03924 [28]. 

Am.
B.
(1997)
14:494-502.
doi:
10.1364/JOSAB.14.0

9. Casado A, Fernández-Rueda A, Marshall TW, Risco-Delgado R, Santos E. Fourth order interference in the Wigner representation for parametric downconversion experiments. Phys Rev A. (1997) 55:3879-90. doi: 10.1103/PhysRevA.55.3879

10. Casado A, Fernández-Rueda A, Marshall TW, Risco-Delgado R, Santos E. Dispersion cancellation and quantum eraser experiments analyzed in the Wigner function formalism. Phys Rev A. (1997) 56:2477-80. doi: 10.1103/PhysRevA.56.2477

11. Casado A, Marshall TW, Santos E. Type II parametric downconversion in the Wigner formalism. Entanglement and Bell's inequalities. J Opt Soc Am B. (1998) 15:1572-7. doi: 10.1364/JOSAB.15.001572

12. Dechoum K, Marshall TW, Santos E. Parametric down and up conversion in the Wigner representation of quantum optics. J Mod Opt. (2000) 47:1273-87. doi: 10.1080/09500340008232173

13. Casado A, Marshall TW, Risco-Delgado R, Santos E. Spectrum of the parametric down converted radiation calculated in the Wigner function formalism. Eur Phys J D. (2001) 13:109-19. doi: 10.1007/s100530170292

14. Casado A, Guerra S, Plácido J. Wigner representation for experiments on quantum cryptography using two-photon polarization entanglement produced in parametric down-conversion. J Phys B Mol Opt Phys. (2008) 41:045501. doi: 10.1088/0953-4075/41/4/045501

15. Casado A, Guerra S, Plácido J. Partial Bell-state analysis with parametric down conversion in the Wigner function formalism. Adv Math Phys. (2010) 2010:501521. doi: 10.1155/2010/501521

16. Casado A, Guerra S, Plácido J. Wigner representation for polarization momentum hyperentanglement generated in parametric down-conversion, and its application to complete Bell-state measurement. Eur Phys J D. (2014) 68:338-48. doi: 10.1140/epjd/e2014-50368-y

17. Casado A, Guerra S, Plácido J. Wigner representation for entanglement swapping using parametric down conversion: the role of vacuum fluctuations in teleportation. J Mod Opt. (2015) 62:377-86. doi: 10.1080/09500340.2014.983571

18. Casado A, Guerra S, Plácido J. Rome teleportation experiment analysed in the Wigner representation: the role of the zeropoint fluctuations in complete one-photon polarization-momentum Bell-state analysis. J Mod Opt. (2018) 65:1960-74. doi: 10.1080/09500340.2018.1478009
19. Weyl H. Quantenmechanik und Gruppentheorie. Z Phys. (1927) 46:1-46. doi: 10.1007/BF02055756

20. Weyl H. The Theory of Groups and Quantum Mechanics. New York, NY: Dover (1931).

21. Wigner EP. On the quantum correction for thermodynamic equilibrium. Phys Rev. (1932) 40:749. doi: 10.1103/PhysRev.40.749

22. Groenewold HJ. On the principles of elementary quantum mechanics. Physica. (1946) 12:405-46. doi: 10.1016/S0031-8914(46) 80059-4

23. Moyal JE. Quantum mechanics as a statistical theory. Proc Cambridge Phil Soc. (1949) 45:99-124. doi: 10.1017/S0305004100000487

24. Hillery M, O'Connell RF, Scully MO, Wigner, EP. Phys Rep. (1984) 106:12168. doi: 10.1016/0370-1573(84)90160-1

25. Zachos CK, Fairlie DB, Curtright TL. Quantum Mechanics in Phase Space. Singapore: World Scientic (2005).

26. Casado A, Fernández-Rueda A, Marshall TW, Martínez J, Risco-Delgado $\mathrm{R}$, Santos E. Dependence on crystal parameters of the correlation time between signal and idler beams in parametric down conversion calculated in the Wigner representation. Eur Phys J D. (2000) 11:465-72. doi: 10.1007/s100530070074

27. Ou ZY, Wang LJ, Mandel L. Evidence for phase memory in two-photon down conversion through entanglement with the vacuum. Phys Rev A. (1990) 41:566. doi: 10.1103/PhysRevA.41.566

28. Santos E. Local realistic interpretation of entangled photon pairs in the Weyl-Wigner formalism. arXiv:1908.03924 (2019).

29. Einstein A. Albert Einstein: Philosopher-Scientist. Schilpp PA, Editor. Evanston: Open Court (1949).

Conflict of Interest: The author declares that the research was conducted in the absence of any commercial or financial relationships that could be construed as a potential conflict of interest.

Copyright (c) 2020 Santos. This is an open-access article distributed under the terms of the Creative Commons Attribution License (CC BY). The use, distribution or reproduction in other forums is permitted, provided the original author(s) and the copyright owner(s) are credited and that the original publication in this journal is cited, in accordance with accepted academic practice. No use, distribution or reproduction is permitted which does not comply with these terms. 